\title{
Fast Computation of the CMH Model
}

\author{
Umesh D. Patel* and Edward Della Torre** \\ *Goddard Space Flight Center, Code 564, Greenbelt, MD 20771 USA, e-mail:upatel@vdragon.gsfc.nasa.gov \\ **George Washington University, DC 20052 USA, e-mail:dellator@seas.gwu.edu
}

\begin{abstract}
A fast difterential equation approach for the DOK model has been extented to the CMH model. Also, a cobweb technique for calculating the CMH model is also presented. The two techniques are calculating the Cintrasted from the point of view of flexibility and computation time.
\end{abstract}

\section{INTRODUCTION}

The $\mathrm{CMH}$ model is a moving Preisach model in which the reversible component of the magnetization is state-dependent. Unlike the DOK model, it accurately predicts the variation in zero-field susceptibility with magnetization. It has not been used much in the past because the DOK model, which is much easier to calculate, is accurate enough in most cases to describe the magnetization process and a fast simple algorithm was not available.

This paper presents two techniques, the differential equation method [1] and the cobweb method [2], which have been previously presented for the DOK model, for calculating the CMH model and contrasts them. In addition an identification strategy for the CMH model will be presented. The speed increase of the two techniques results from computing the magnetization by simply adding the change in magnetization due to any change in applied field to the previously computed magnetization, instead of integrating over the Preisach plane at each step of the computation.

The differential equation method calculates the susceptibility in closed form for Gaussian Preisach functions. This limits the technique to simple magnetization distributions that are integrable. However, it is a variable step-size technique that is not limited by the discretization error in the magnetization and distributes pseudo-hysterons over the two hysterons switching at the same time, leading to a smooth magnetization curve. Changing the error criterion during a calculation is difficult in the midst of a calculation.

\section{THE DIFFERENTIAL EQUATION METHOD}

We assume that the Preisach function of the $\mathrm{CMH}$ model is the standard moving model of the form

$$
P\left(h_{1}, h_{k}\right)=\frac{M_{1}}{2 \pi \sigma_{i} \sigma_{k}} \exp \left[-\frac{\left(h_{k}-\bar{h}_{k}\right)^{2}}{2 \sigma_{k}^{2}}-\frac{\left(h_{1}+\alpha M\right)^{2}}{2 \sigma_{i}^{2}}\right]
$$

We compute the susceptibility using

$$
\chi=M_{s}\left[S \chi_{1}+(1-S) \chi_{R}\right]
$$

where $\chi_{1}$ is the susceptibitity of the irreversible component, $\chi_{R}$ is the susceptibility of the reversible component and $S$ is the squareness of the material. In the CMH model, $\chi_{R}$ is given by

$$
\chi_{R}=\frac{\partial m_{R}}{\partial a_{+}} \frac{d a_{+}}{d h}+\frac{\partial m_{R}}{\partial a_{-}} \frac{d a_{-}}{d h}+\frac{\partial m_{R}}{\partial H} \frac{d H}{d h}+\frac{\partial m_{R}}{\partial h}
$$

In the following analysis, we will use

$$
m_{R}=a_{+} f(H)+a_{-} f(H),
$$

where

$$
f(H)=1-e^{-\not H} .
$$

It can be shown that

$$
a_{ \pm}=\iint_{Q= \pm 1} \exp \left(\mp \gamma h_{i}\right) p\left(h_{k}, h_{i}\right) d h_{k} d h_{i} .
$$

From [1] the irreversible susceptibility for increasing operative field is given by

$$
\begin{array}{r}
x_{I}=\frac{1}{\sigma \sqrt{2 \pi}} e^{\frac{\left(h-h_{k}\right)^{2}}{2 \sigma^{2}}}\left[\operatorname{erf}\left(\frac{(1+\lambda) h+\kappa \bar{h}_{k}}{\tau \sqrt{2}}\right)\right. \\
\left.-\operatorname{erf}\left(\frac{h_{1}+\lambda h+\kappa \bar{h}_{k}}{\tau \sqrt{2}}\right)\right]
\end{array}
$$

and for decreasing operative field is given by

$$
\begin{array}{r}
\chi_{I}=\frac{1}{\sigma \sqrt{2 \pi}} e^{-\frac{\left(h-h_{k}\right)^{2}}{2 \sigma^{2}}}\left[\operatorname{erf}\left(\frac{h_{1}+\lambda h-\kappa \bar{h}_{k}}{\tau \sqrt{2}}\right)\right. \\
\left.-\operatorname{erf}\left(\frac{(1+\lambda) h-\kappa \bar{h}_{k}}{\tau \sqrt{2}}\right)\right]
\end{array}
$$

Using (1) and (6), we can derive $d a_{+} / d h$ for increasing operative field as

$$
\begin{aligned}
\frac{d a_{+}}{d h}=\frac{e^{\left(\gamma^{2} \sigma_{i}\right)}}{2 \sigma \sqrt{2 \pi}} e^{\frac{\left(h-h_{k}+x \sigma_{i}\right)^{2}}{2 \sigma^{2}}}\left[\operatorname{erf}\left(\frac{(1+\lambda) h+\kappa \bar{h}_{k}+\frac{1}{2} \tau^{2} \gamma}{\tau \sqrt{2}}\right)\right. \\
-e r f\left(\frac{h_{1}+\lambda h+\kappa \bar{h}_{k}+\frac{1}{2} \tau^{2} \gamma}{\tau \sqrt{2}}\right)
\end{aligned}
$$

and for decreasing operative field as

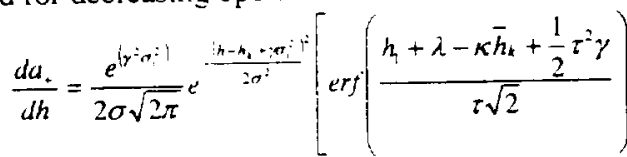

$$
\begin{aligned}
& -e r f\left(\frac{(1+\lambda) h-\kappa \bar{h}_{k}+\frac{1}{2} \tau^{2} \gamma}{\tau \sqrt{2}}\right)
\end{aligned}
$$

Similarly, we can derive daidh for increasing operative field as 


$$
\begin{aligned}
\frac{d a}{d h}=\frac{e^{\mid \gamma+r}}{2 \sigma \sqrt{2 \pi}} \cdot a^{*} & {\left[\frac{-\left(1+\lambda h-\kappa \bar{h}_{k}+\frac{1}{2} \tau^{2} \gamma\right.}{\tau \sqrt{2}}\right) } \\
& -\operatorname{erf}\left(\frac{-h_{1}-\lambda h-\kappa \bar{h}_{k}+\frac{1}{2} \tau^{2} \gamma}{\tau \sqrt{2}}\right)
\end{aligned}
$$

and for decreasing field as

$$
\begin{aligned}
& \frac{d a_{-}}{d h}=\frac{e^{\left(\gamma^{2} \sigma_{i}^{2}\right)}}{2 \sigma \sqrt{2 \pi}} e^{\frac{\left(h-h_{1}+\pi_{t}^{2}\right)}{2 \sigma^{2}}}\left[\operatorname{erf}\left(\frac{-h_{1}-\lambda h+\kappa \bar{h}_{1}+\frac{1}{2} \tau^{2} \gamma}{\tau \sqrt{2}}\right)\right. \\
& -e r f\left(\frac{-(1+\lambda) h+\kappa \vec{h}_{k}+\frac{1}{2} \tau^{2} \gamma}{\tau \sqrt{2}}\right)
\end{aligned}
$$

The parameters $\lambda, \kappa$, and $\tau$ are the same as in [1]. The reversible susceptibility is given by

$$
\begin{array}{r}
a_{+} f(H)-a_{-} f(H)+\left(a_{+} f^{\prime}(H)-a_{-} f(-H)\right) \\
-\alpha S M_{S} \chi_{l}\left(a_{+} f^{\prime}(H)-a_{-} f(-H)\right)
\end{array}
$$

The sum of (7) and (13) gives the total susceptibility and then the next magnetization can be computed by

$$
M(H+\Delta H)=m(H)+\chi \Delta H
$$

There are several problems with this technique. The first problem is that the stability of this method requires small step in $\mathrm{H}$ under certain conditions. The second problem is that when the reversible magnetization is close to saturation the irreversible magnetization can get to be very large due to the exponential increase of $f(H)$. This can be overcome by setting the magnetization to saturation when it gets close to saturation. However, this puts a glitch in the solution which may be undesirable.

\section{THE COBWEB METHOD}

The cobweb grid in Preisach plane was developed to achieve higher speed and accuracy of numerical computation for irreversible magnetization [2]. Here, the $\mathrm{CMH}$ model is implemented based on the cobweb grid systems for irreversible magnetization and $a_{+}$and $a$ variables for the reversible magnetization.

The cobweb method distributes hysterons uniformly in $\theta$ in the interval $(0,2 \pi)$ and in $\rho$ in $(0,1)$ on $m \times n$ grid. The relationship between these co-ordinates and the operative coordinates $h_{i}$ and $h_{k}$ for irreversible magnetization are given by

$$
\begin{aligned}
& h_{k}=\bar{h}_{k}+\sigma_{k} \sin \theta \sqrt{-2 \log (1-\rho)} \\
& h_{i}=\sigma_{i} \cos \theta \sqrt{-2 \log (1-\rho)}
\end{aligned}
$$

The contribution of any single hysteron to the total moment is equal to that of all the others and is equal to $h_{s} / m n$. Thus, the maximum error in $M$ is half that and by choosing $m n$ large enough the error can be made arbitrarily small
Similarly, the operative co-ordinates $h_{\text {, and }} h_{k}$ for $a$, are given by

$$
\begin{aligned}
& h_{k}=\bar{h}_{k}+\sigma_{k} \sin \theta \sqrt{-2 \log (1-\rho)} \\
& h_{i}=-\gamma \sigma_{i}^{2}+\sigma_{i} \cos \theta \sqrt{-2 \log (1-\rho)}
\end{aligned}
$$

and for $a$. the operative co-ordinates are given by

$$
\begin{aligned}
& h_{k}=\bar{h}_{k}+\sigma_{k} \sin \theta \sqrt{-2 \log (1-\rho)} \\
& h_{i}=\gamma \sigma_{i}^{2} \sigma_{1} \cos \theta \sqrt{-2 \log (1-\rho)}
\end{aligned}
$$

The contribution of any single hysteron to the total $a_{+}$or $a$. is equal to $\exp \left(\gamma^{2} \sigma^{2}, 2\right) / m n$.

\section{MODEL PARAMETER IDENTIFICATION}

An identification method will be presented for the CMH model which is a modification of the DOK identification algorithm [3]. All parameters are computed in essentially the same way as in the DOK model except for $\gamma$. In the DOK model $\gamma$ is the reversible zero-field susceptibility that is independent of the magnetic state. Since the reversible susceptibility for zero field is now a function of magnetization. Thus, we recommend identification of $\gamma$ by fitting the descending major loop near positive saturation.

\section{DISCUSSION AND CONCLUSION}

In previous models, the squareness $S$ and were the same for the entire distribution. This made it difficult to satisfy the crossover condition for particles with large hi. In the cobweb model it is possible to decrease $S$ or for particles with large hi.

We have presented two fast techniques for computing the magnetization expected for the $\mathrm{CMH}$ model, both of which produce the same results and are fast. We will present results for both methods of computation.

\section{ACKNOWLEDGEMENTS}

We would like to thank Ann Reimers and Shailendra Garg for useful discussions.

\section{REFERENCES}

[1]. A. Reimers and E. Della Torre, "Fast Preisach based magnetization model and fast inverse hysteresis model," IEEE Trans. Magn., 34, Nov. 1998, pp.3857-3866.

[2]. O. Alejos and E. Della Tore, "Improving numerical simulations of Preisach models for accuracy and speed," presented at INTERMAG, Toronto. Canadia, April 9-14, 2000. IEEE Trans. Magn., (in press).

[3]. E. Della Torre, "Parameter identification of completemoving hysteresis model using major loop data," IEEE Trans. Huzn.. 30, Nov. 1994, pp.4987-5000. 